\title{
A Novel Megastable Oscillator with a Strange Structure of Coexisting Attractors: Design, Analysis, and FPGA Implementation
}

\author{
Kui Zhang, ${ }^{1}$ M. D. Vijayakumar, ${ }^{2}$ Sajjad Shaukat Jamal $\left(\mathrm{D},{ }^{3}\right.$ Hayder Natiq, ${ }^{4}$ \\ Karthikeyan Rajagopal $\left(\mathbb{D},{ }^{5}\right.$ Sajad Jafari, ${ }^{6,7}$ and Iqtadar Hussain ${ }^{8}$ \\ ${ }^{1}$ School of Electronic Engineering, Changzhou College of Information Technology, Changzhou 213164, China \\ ${ }^{2}$ Center for Materials Research, Chennai Institute of Technology, Chennai, India \\ ${ }^{3}$ Department of Mathematics, College of Science, King Khalid University, Abha, Saudi Arabia \\ ${ }^{4}$ Information Technology Collage, Imam Ja'afar Al-Sadiq University, Baghdad 10001, Iraq \\ ${ }^{5}$ Center for Nonlinear Systems, Chennai Institute of Technology, Chennai 600069, Tamilnadu, India \\ ${ }^{6}$ Health Technology Research Institute, Amirkabir University of Technology, 424 Hafez Ave., Tehran 15875-4413, Iran \\ ${ }^{7}$ Department of Biomedical Engineering, Amirkabir University of Technology, 424 Hafez Ave., Tehran 15875-4413, Iran \\ ${ }^{8}$ Department of Mathematics, Statistics and Physics, Qatar University, Doha 2713, Qatar
}

Correspondence should be addressed to Karthikeyan Rajagopal; rkarthiekeyan@gmail.com

Received 7 June 2021; Accepted 19 August 2021; Published 27 August 2021

Academic Editor: Ahmed A. Abd El-Latif

Copyright (๑) 2021 Kui Zhang et al. This is an open access article distributed under the Creative Commons Attribution License, which permits unrestricted use, distribution, and reproduction in any medium, provided the original work is properly cited.

\begin{abstract}
Megastable chaotic systems are somehow the newest in the family of special chaotic systems. In this paper, a new megastable two-dimensional system is proposed. In this system, coexisting attractors are in some islands, interestingly covered by megalimit cycles. The introduced two-dimensional system has no defined equilibrium point. However, it seems that the origin plays the role of an unstable equilibrium point. Therefore, the attractors are determined as hidden attractors. Adding a forcing term to the system, we can obtain chaotic solutions and coexisting strange attractors. Moreover, the effect of three different values of the forcing term's amplitude is studied. The dynamical properties of the designed system are investigated using attractor plots, bifurcation diagrams, and Lyapunov Exponents diagram. Phase portraits of the novel megastable oscillator are presented by FPGA design. Xilinx system generator block diagrams of the proposed system and trigonometric functions are also presented.
\end{abstract}

\section{Introduction}

Finding new special chaotic systems or, more specifically, new systems with special and unique dynamical characteristics has been an active area of research since about 30 years ago. First, Sprott has introduced some elegant quadratic three-dimensional chaotic systems [1]. Then, people have tried to find the simplest cases of special chaotic systems [2]. For example, chaotic systems with many wings have been designed [3], simplest jerk systems have been introduced [4], elegant hyperchaotic systems have been found $[5,6]$, circulant chaotic systems have been constructed [7], and symmetric chaotic flows have been investigated $[8,9]$.

One crucial point about dynamical systems is the role of equilibria in them. It was believed that the strange attractors and unstable equilibrium points have a strong relationship. More particularly, unstable equilibrium points were supposed to be the clue for strange attractors. However, finding dissipative chaotic systems with no equilibria was an exciting discovery which challenged that confidence [10]. Also, chaotic systems with stable equilibria changed many conventional beliefs about the reason for the creation of strange attractors [11]. Systems 
with lines, curves, and surfaces of equilibria came one after another and shed more light on many unknown points in the field. Nevertheless, calculating system's equilibrium points is the first basic step of analyzing its dynamics.

Multistability is an important phenomenon in dynamical systems [12], which is a kind of double-edged sword feature. While it can cause unwanted shifts in a system's dynamic, it can provide extra flexibility, e.g., for the control aims. Sometimes the number of coexisting attractors in a multistable system becomes infinite. In such a scenario, if those infinite attractors are uncountable, the system is called extreme multistable [13-15]. Initial conditions play the role of bifurcation parameters in such systems. However, when those infinite attractors are countable, the system is called megastable. The term "megastable" was first used in [16]. Megastable chaotic systems are somehow the newest in the family of special chaotic systems [16]. In summary, the main difference between a megastable system and an extreme multistable system is in the countability of the system's coexisting attractors. In both terms, the number of coexisting attractors is limitless. Many interesting configurations of coexisting attractors have been reported in megastable systems [17].

Hidden and self-excited are types of attractors. Many research studies have focused on categorizing dynamical attractors based on them [18-20]. A self-excited attractor can be detected easily by observing an unstable equilibrium point in the attractor's basin of attraction. However, an attractor with no equilibrium point inside its basin of attraction is called hidden [21].

The analysis of the dynamics of a dynamical system needs some powerful tools to provide primary information about the system behaviors in different conditions. In this way, obtaining the bifurcation diagram is considered as one of the primary steps of analyzing system's dynamics. Another popular tool for analyzing the dynamics of a system is the Lyapunov Exponents spectrum (LE diagram). LE is simply a quantitative measure that can prove the presence of chaos in a dynamical system [2].

Chaotic systems have many engineering applications. They can be used in image encryption [22], communication [23], circuits [24], robots [25], and so on [26]. Field programmable gate array (FPGA) implementation of nonlinear systems plays a vital role in realizing a system using targeted hardware. In fact, FPGAs are a kind of chips or gate arrays that are easy to program. Engineering applications of FPGA are wired and wireless communication, industrial and medical systems, military, and aerospace. FPGAs are costeffective depending on their families, such as Spartan, Kintex, and Virtex. Many researchers have shown interest in FPGA implementation of chaotic systems. They have performed the software-hardware interface by implementing chaotic systems in FPGA [27].

In this paper, a new two-dimensional megastable system is proposed. The sections of this paper are arranged as follows. The new proposed two-dimensional system is introduced in Section 2. Moreover, the dynamical properties are explained in that section. Next, Section 3 describes the FPGA implementation of the proposed system. The conclusion of the paper is presented in Section 4.

\section{A New Megastable Chaotic Oscillator}

Consider System (1), which is a two-dimensional nonlinear autonomous oscillator,

$$
\begin{aligned}
& \dot{x}=-0.1 y+x \frac{\cos (r)}{r}, \\
& \dot{y}=\sin (0.1 x)+y \frac{\cos (r)}{r}, \\
& r=\sqrt{x^{2}+y^{2}}
\end{aligned}
$$

System (1) is symmetric around the origin because the equations are invariant under the transformation $(x, y) \longrightarrow(-x,-y)$.

System (1) has no equilibrium points since no points can be found to solve the equations $-0.1 y+x(\cos (r) / r)=0$ and $\sin (0.1 x)+y(\cos (r) / r)=0$. However, the origin $(0,0)$ mimics an unstable equilibrium. This system is megastable since it has infinite countable coexisting attractors (here, limit cycles).

Figure 1 is a plot of coexisting limit cycles in System (1) resulted from random initial conditions distributed around the $x$-axis. The formation of these attractors is noticeable. We can see islands of attractors consisting of 3, 4, and 5 limit cycles. Surprisingly 11 islands are enclosed by a huge limit cycle. Due to the attractors' isolated configuration, the phrase "islands of attractors" can be used for such systems attractors. Figure 1 includes both transient and final states of the trajectories to show the areas of islands more significantly.

By introducing a periodic external force in the first equation of System (1), the following forced oscillator is achieved:

$$
\begin{aligned}
& \dot{x}=-0.1 y+x \frac{\cos (r)}{r}+A \sin (\omega t), \\
& \dot{y}=\sin (0.1 x)+y \frac{\cos (r)}{r}, \\
& r=\sqrt{x^{2}+y^{2}} .
\end{aligned}
$$

It is primarily desired to find chaos in System (2). Many sets of $(A, \omega)$ may result in chaos. By trial and error, $\omega=0.6$ is chosen, and $A$ is considered as the bifurcation parameter. However, an infinite number of coexisting attractors are detected. Each attractor can go through different dynamical regimes during the change in the bifurcation parameter. Thus, two attractors (one around the origin and the other around the point $(60,0))$ are selected, and their occurring bifurcations are tracked to show such a difference.

Figure 2 shows the bifurcation diagram and LEs diagram versus $A$ for the nearest attractor around the origin resulted from the constant initial conditions $(0.1,0)$. It is seen that the dynamical solution starts from an attracting torus (one negative and two zero LEs). After observing limit cycles (two negative and one zero LEs), chaos occurs (one positive LE). Then, the dynamic alternates between chaos and limit cycles. 


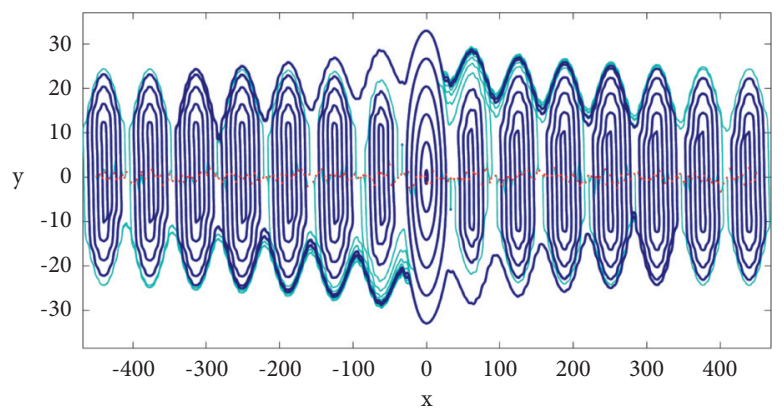

Figure 1: Coexisting limit cycles in System (1) resulted from random initial conditions distributed around the $x$-axis. Islands of attractors can be observed, consisting of 3, 4, and 5 limit cycles. Surprisingly 11 islands are enclosed by a huge limit cycle. The transients are shown in cyan, and steady states are shown in dark blue.

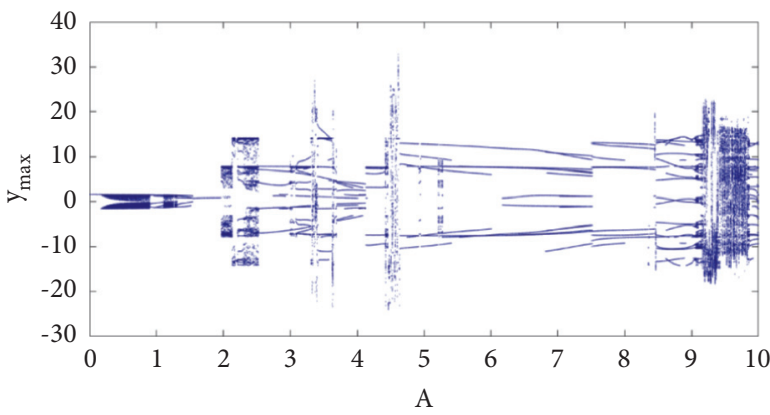

(a)

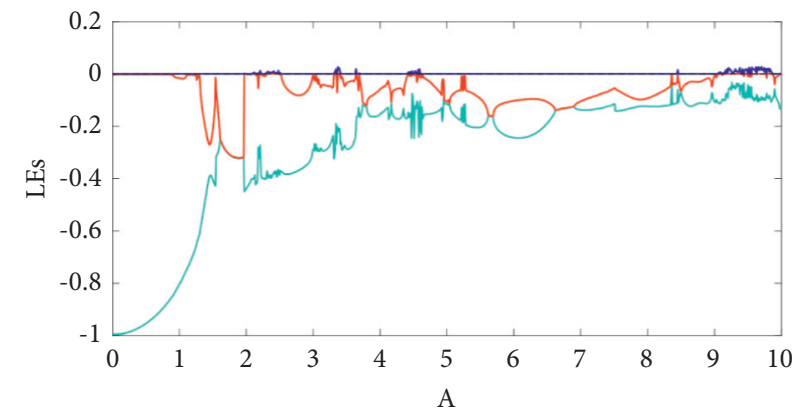

(b)

Figure 2: (a) Local maximum values of " $y$ " time-series versus $A$ in System (2) when $\omega=0.6$. The initial conditions are $(0.1,0)$ without reinitiating. Thus, the diagram is only related to the first (inner) limit cycle around the origin. (b) Corresponding Lyapunov exponent diagram. The dynamic of the system starts from an attracting torus, then limit cycles observed, and finally strange attractors appear.

Figure 3 shows the bifurcation diagram and LEs diagram versus $A$ for the nearest attractor around the point $(60,0)$ resulted from the constant initial conditions $(60,0)$. It is seen that the dynamical solutions are different from the previous attractor. It starts from a limit cycle and continues with it, encountering narrow areas of chaotic attractor. It occasionally has an attracting torus in larger values of the parameter.

It should be mentioned that the LEs represented in Figures 2 and 3 are plotted and calculated using the Wolf algorithm [28] with the run time of 2000. Moreover, these two bifurcation diagrams and LEs diagrams can help comprehend the system behaviors in two different initial conditions. Furthermore, the local maxima of the time-series of variable $y\left(y_{\max }\right.$, which are the peaks of the time-series of $y$ ) are considered for plotting the bifurcation diagrams of the proposed system.

Figure 4 shows coexisting attractors for different values of the amplitude of the forcing term. While the system can have different types of attractors (limit cycle, torus, and strange attractor) simultaneously, increasing the amplitude makes them become closer and even overlap with each other.

\section{FPGA Implementation of Novel Megastable Oscillator}

FPGAs are gate arrays that are programmable, and they can be designed to meet a special need. FPGAs are also costefficient, and they are simple to design, implement, and fast prototyping. Some of the recent pieces of the literature on FPGA design had attracted many researchers, such as variable-order fractional operator [29], hardware implementation of the multistable chaotic jerk system [30], FPGA implementation of self-excited and hidden chaotic systems [31], the discrete memristor chaotic system realized using hardware [32], and digital implementation of the memristive chaotic circuit [33]. Development of the nonlinear system on an FPGA using VHDL or VERILOG hardware description language is very work-intensive. It is easy to design the system using the Xilinx system generator rather than writing test benches for the VHDL or Verilog HDL programming. In a Simulink library browser, a separate Xilinx block set toolbox is readily available to design the system in the Xilinx system generator platform. Simulink diagrams of Systems (1) and (2) are shown in Figures 5 and 6 using Xilinx system generator software. Basic blocks such as adder, subtractor, multiplier, divider, constant multiplier, and square root are used to design the proposed system in FPGA. All Xilinx block sets are different from MATLAB Simulink blocks with the Xilinx logo in them. Additional blocks are created to represent trigonometric functions present in the proposed system. By applying the Taylor series (equations (3) and (4)), trigonometric functions are implemented using the readily available (XSG) Xilinx System Generator block sets, which is shown in Figure 7 (sine function) and Figure 8 (cosine function). All these blocks used to design the proposed 


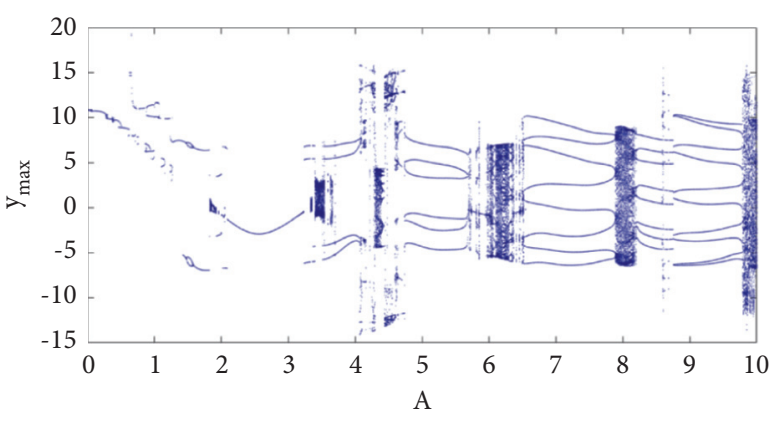

(a)

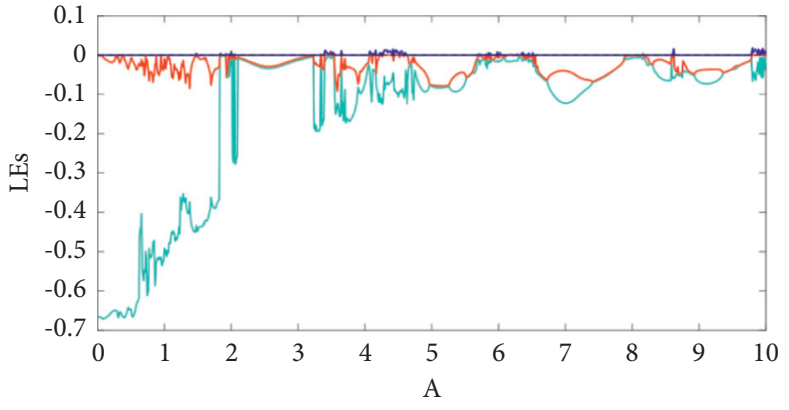

(b)

Figure 3: (a) Local maximum values of " $y$ " time-series versus $A$ in System (2) when $\omega=0.6$. The initial conditions are (60,0) without reinitiating. Thus, the diagram is only related to the inner limit cycle around the point $(60,0)$. (b) Corresponding Lyapunov exponent diagram. The dynamic of the system starts from a limit cycle, and then narrow areas of chaos are observed.

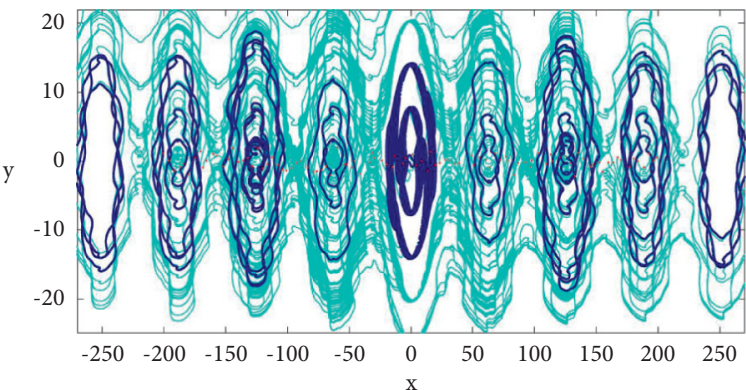

(a)

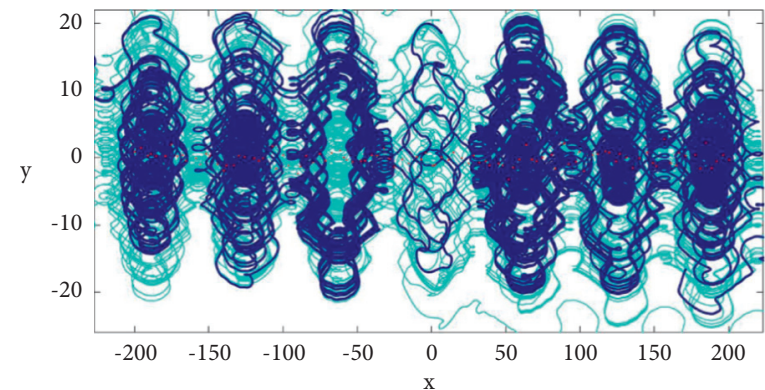

(b)

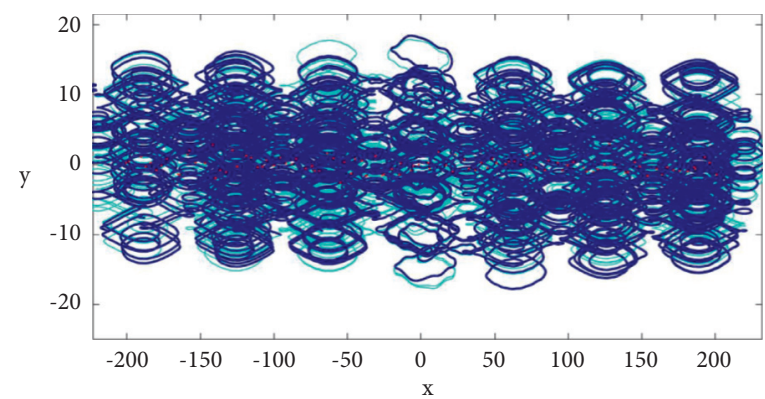

(c)

Figure 4: Coexisting attractors in System (2) resulted from random initial conditions distributed around the $x$-axis. The transients are shown in cyan, and steady states are shown in dark blue. The frequency of the forcing term is $\omega=0.6$, and the amplitude is (a) $A=2$, (b) $A=4.3$, and (c) $A=9.7$. Increasing the amplitude of the forcing term leads to more overlapped islands.

system are configured according to the IEEE754 standard, and the step size $h=0.01$ is set. Integrator blocks of the state equations are designed using Forward Euler's Method, and the mathematical equation to design integrators is expressed in equation (5). A set of discretized system equations are stated in equations (6) and (7).

$$
\begin{aligned}
\sin (r) & =-\frac{r^{3}}{3 !}+\frac{r^{5}}{5 !}-\frac{r^{7}}{7 !}+\frac{r^{9}}{9 !}-\frac{r^{11}}{11 !}+\frac{r^{13}}{13 !}-\cdots \\
\cos (r) & =1-\frac{r^{2}}{2 !}+\frac{r^{4}}{4 !}-\frac{r^{6}}{6 !}+\frac{r^{8}}{8 !}-\frac{r^{10}}{10 !}+\frac{r^{12}}{12 !}-\cdots, \\
k_{n+1} & =k_{n}+h f\left(k_{n-1}\right),
\end{aligned}
$$




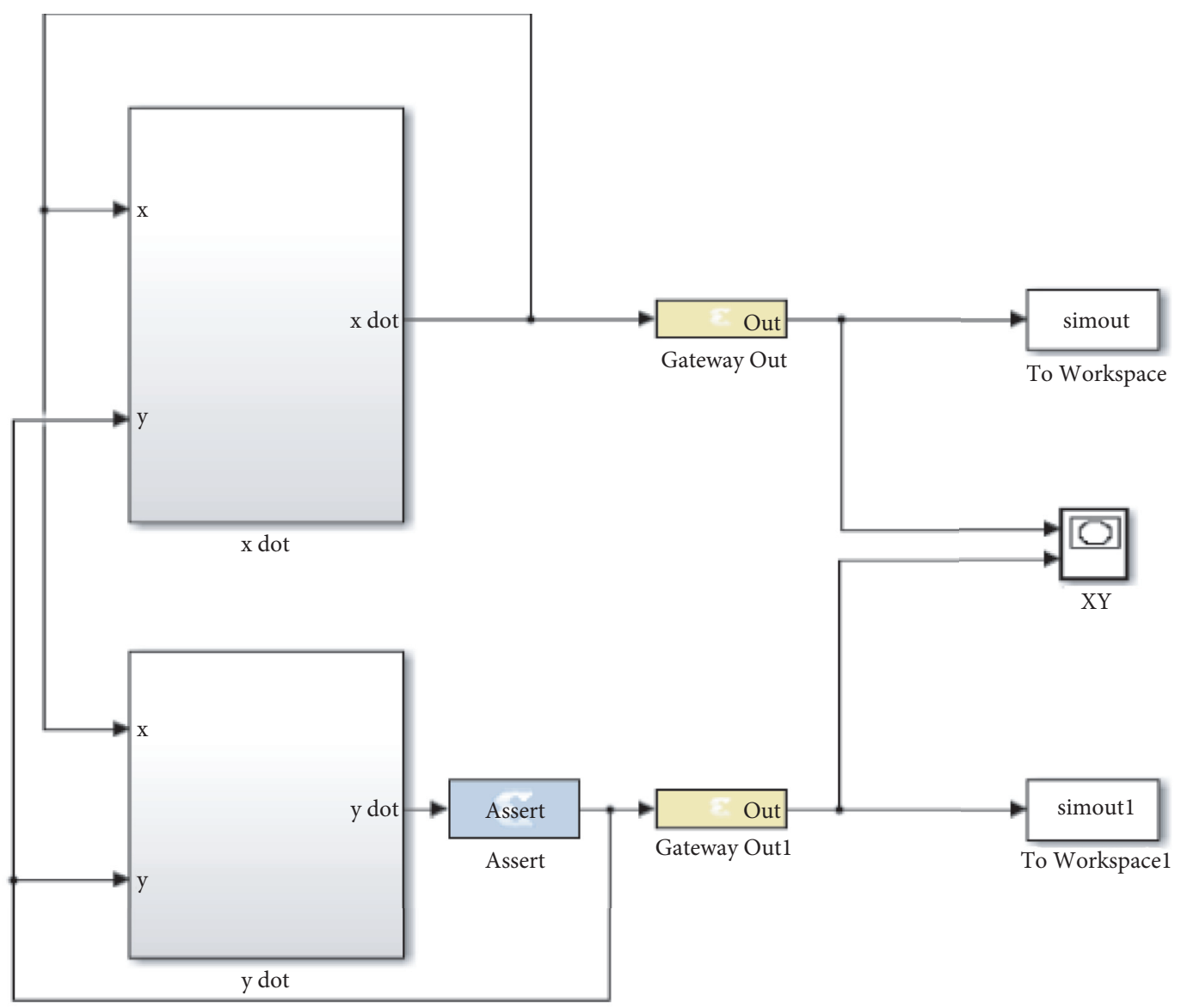

FIGURE 5: Xilinx system generator (XSG) Simulink diagram of System (1). It is another representation of the proposed system in autonomous form.

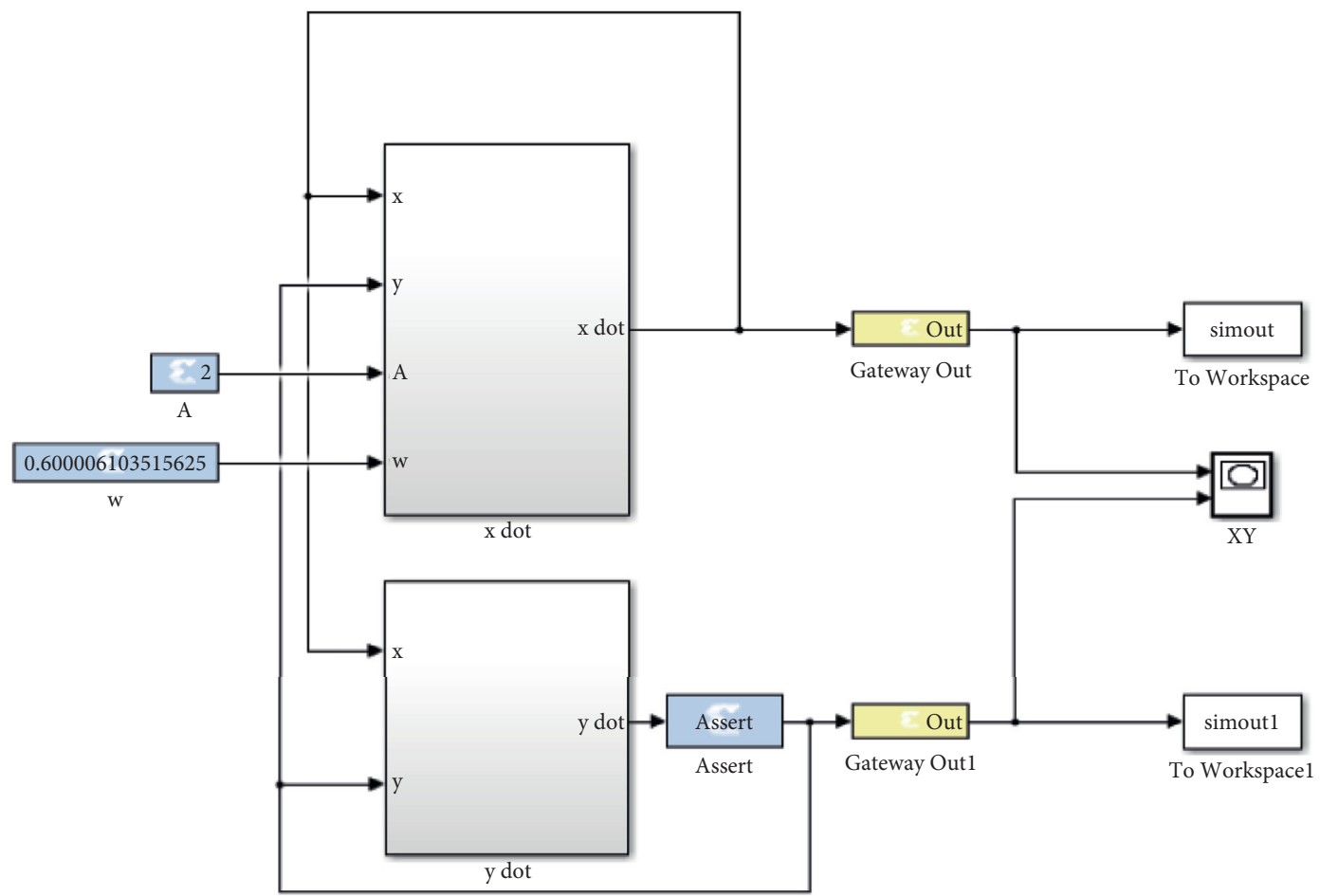

FIGURE 6: Xilinx system generator (XSG) Simulink diagram of System (2) with $\omega=0.6$ and amplitude $A=2$. It is another representation of the proposed system in nonautonomous form. 


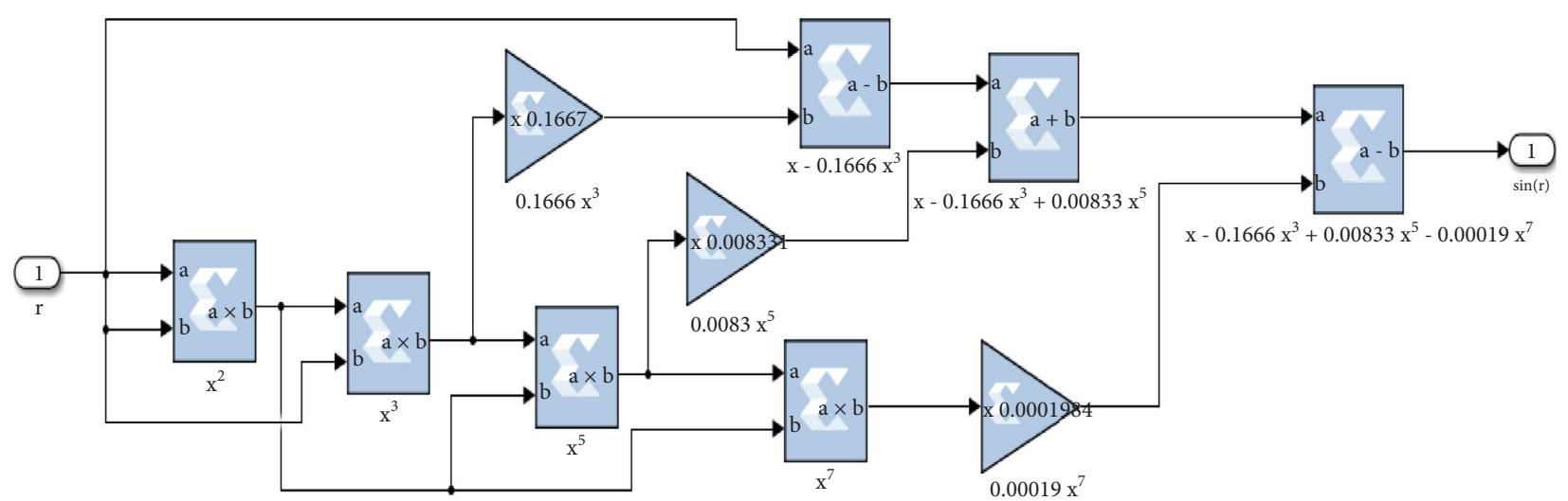

FIGURE 7: Taylor series of sine function implemented using (XSG) Xilinx system generator toolbox. It is discretized implementation of sine function based on equation (3).

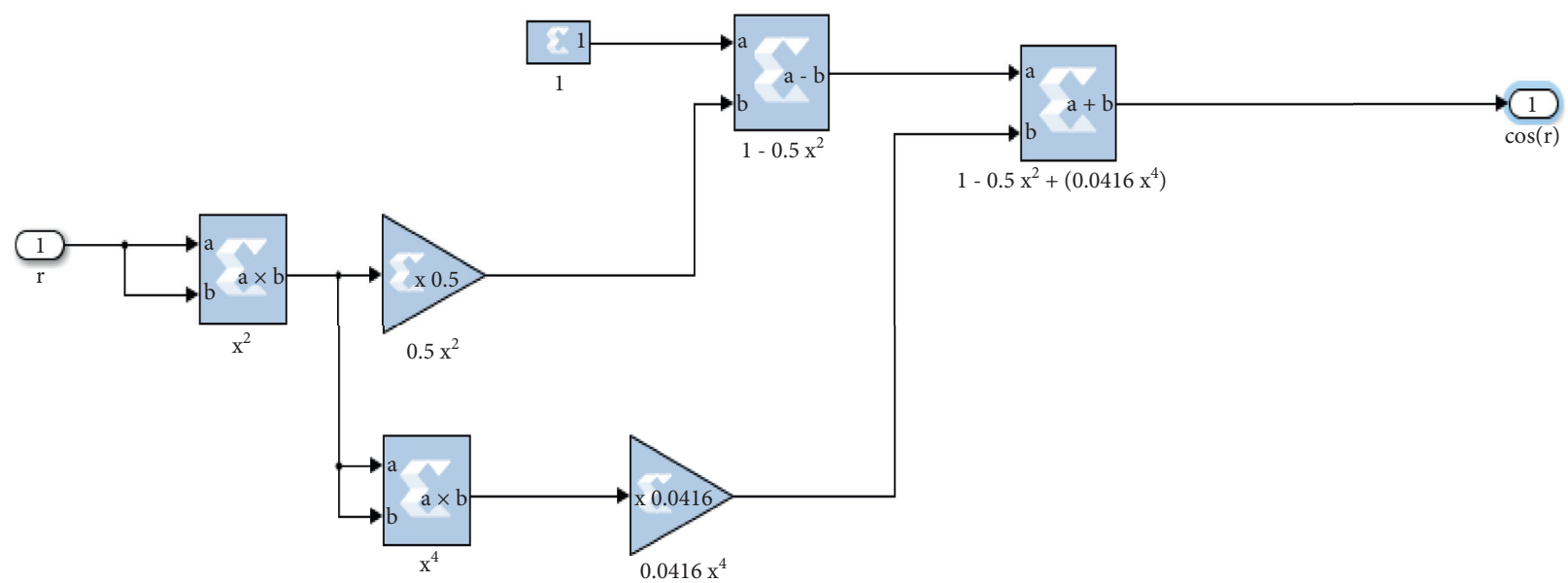

FIGURE 8: Taylor series of cosine function implemented using (XSG) Xilinx system generator toolbox. It is discretized implementation of cosine function based on equation (4).

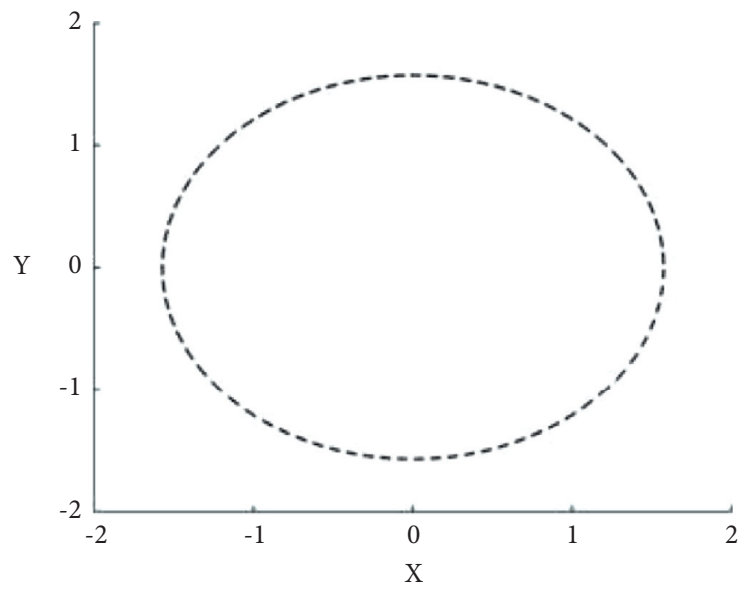

(a)

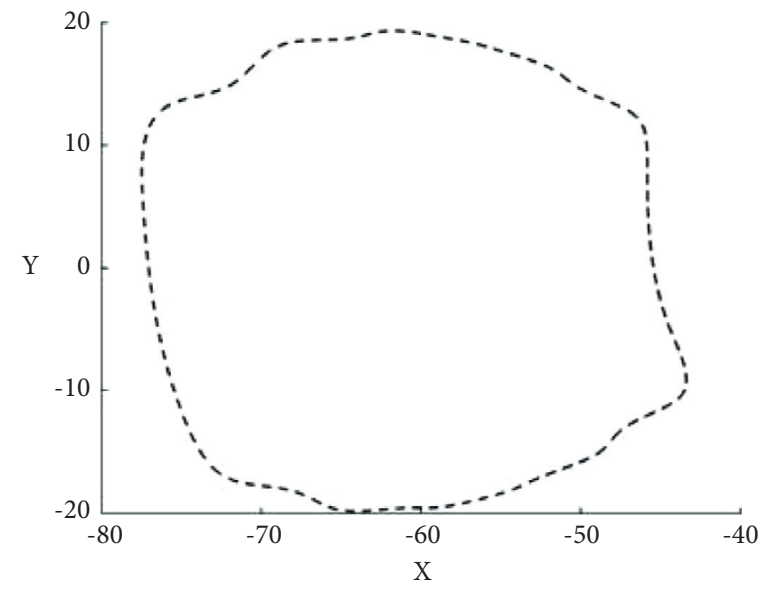

(b)

FIgURE 9: Continued. 


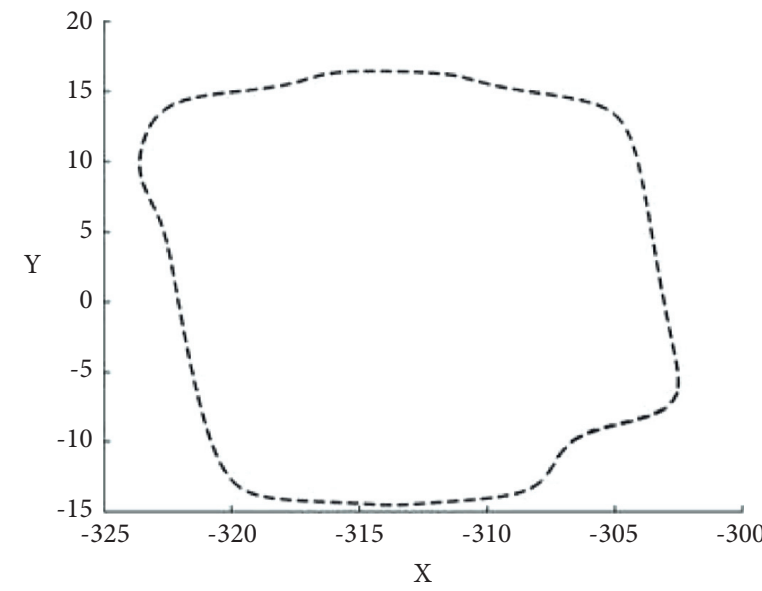

(c)

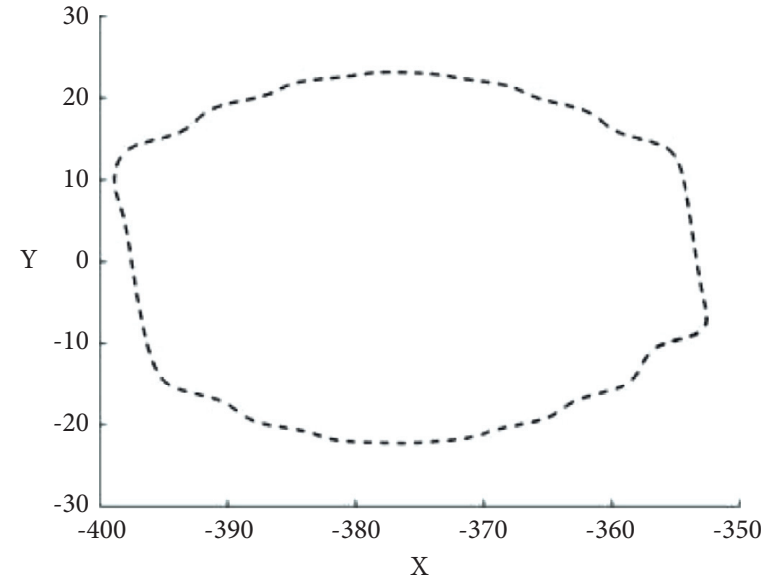

(d)

Figure 9: Phase portraits in the plane $(x, y)$ for System (1) illustrating megastability obtained by considering random initial conditions distributed around the $x$-axis. For all initial conditions, the final state represents a limit cycle.

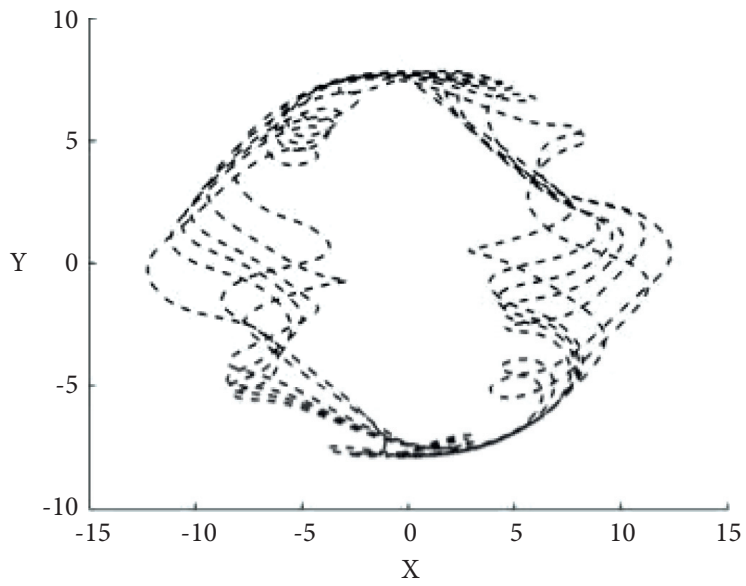

(a)

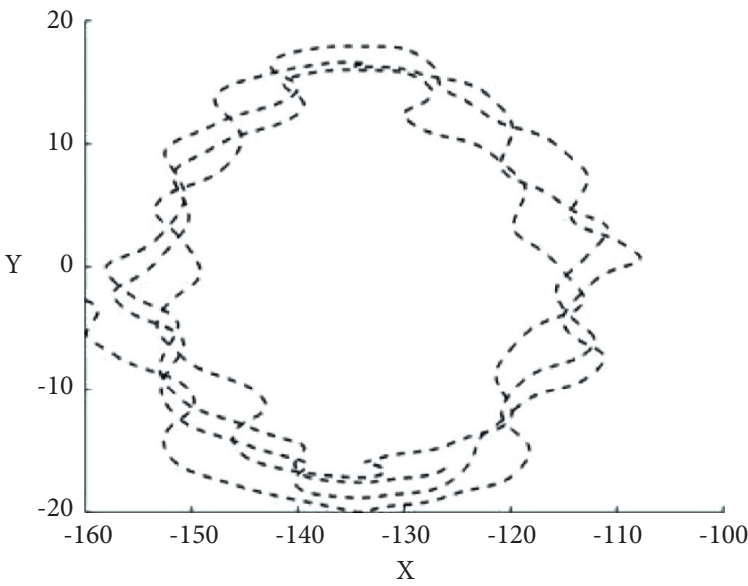

(c)

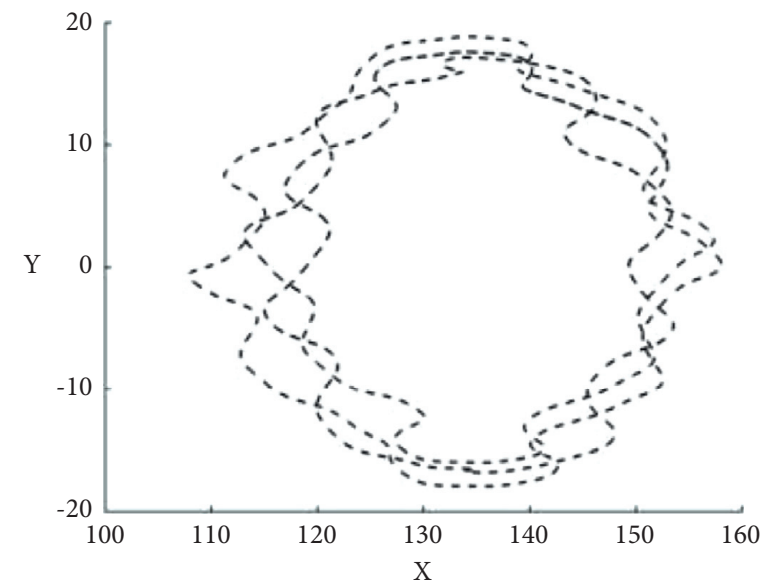

(b)

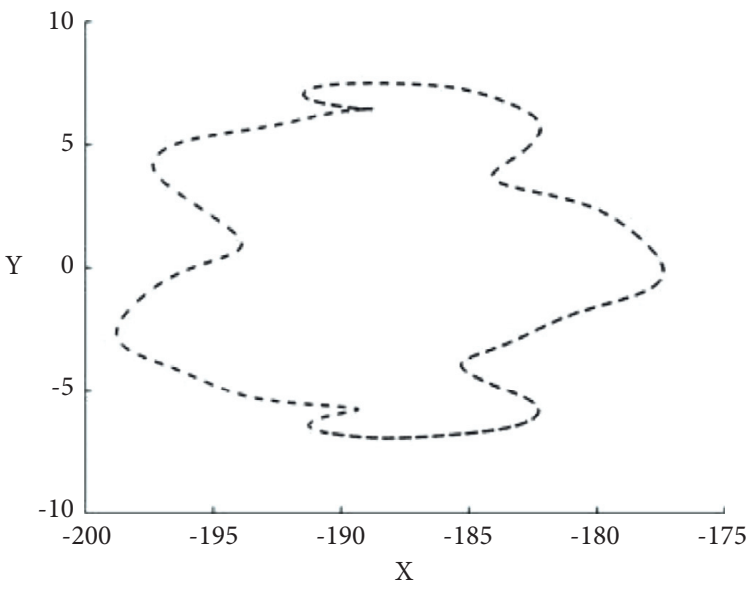

(d)

FIGURE 10: Phase portrait in the plane $(x, y)$ for System (2) illustrating megastability obtained by considering random initial conditions distributed around the $x$-axis. The frequency of the forcing term is $\omega=0.6$, and the amplitude $A=2$. Adding a forcing term can make the system exhibit various dynamics. 


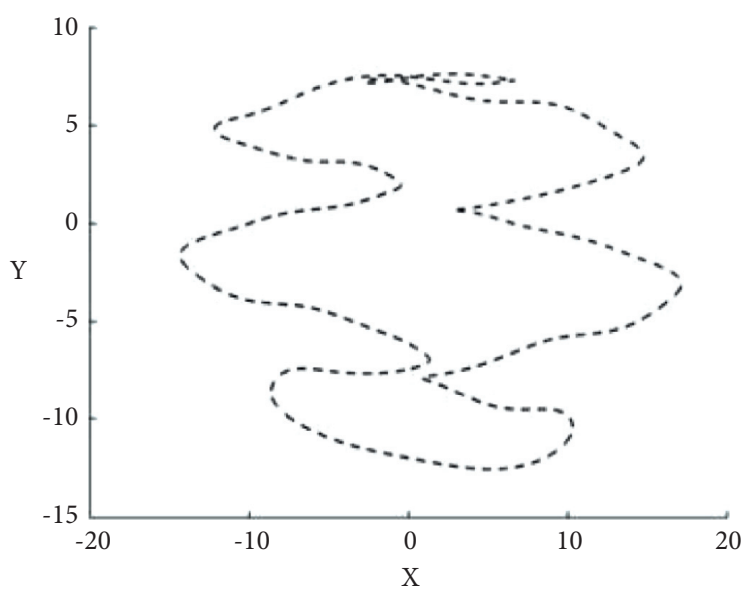

(a)

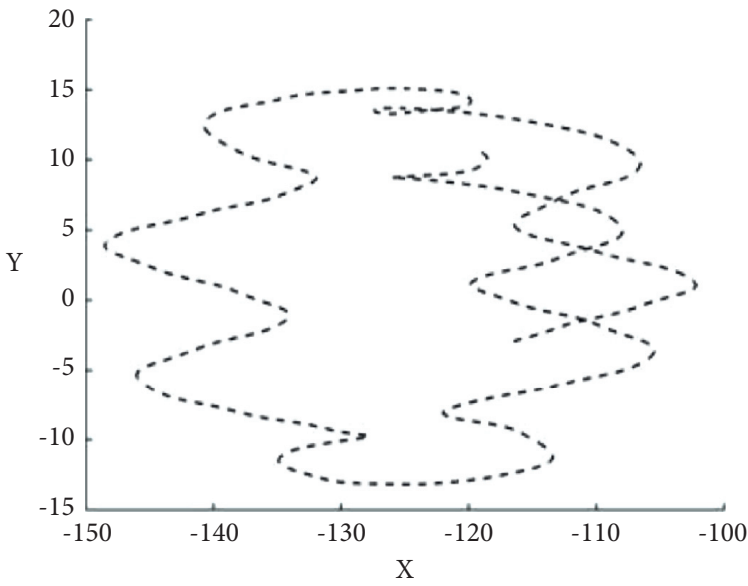

(c)

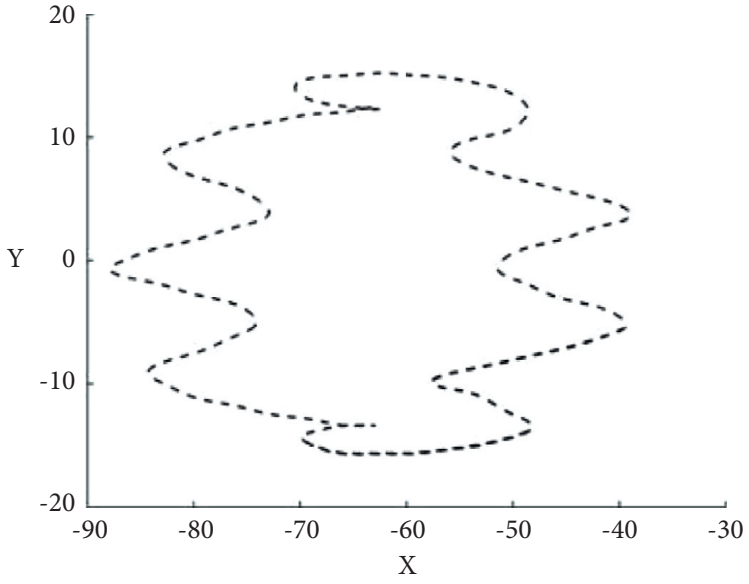

(b)

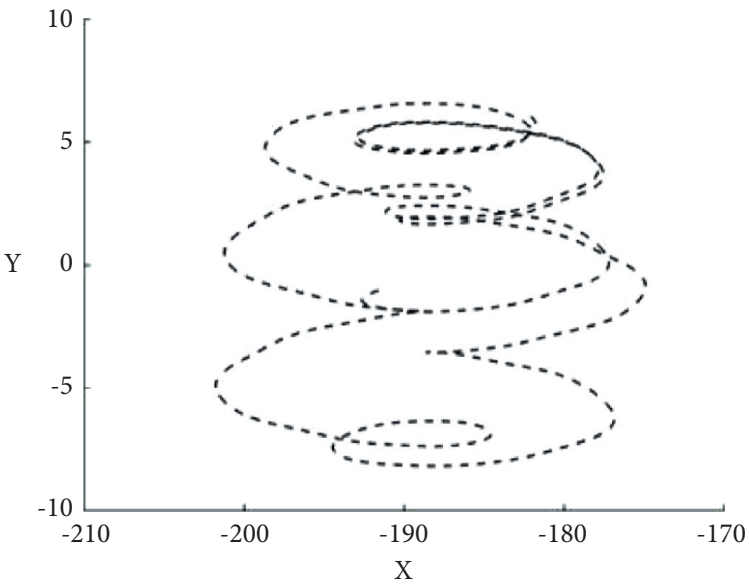

(d)

FIgURE 11: Phase portrait in the plane $(x, y)$ for System (2) illustrating megastability obtained by considering random initial conditions distributed around the $x$-axis. The frequency of the forcing term is $\omega=0.6$, and the amplitude $A=4.3$. Adding a forcing term can make the system exhibit various dynamics.

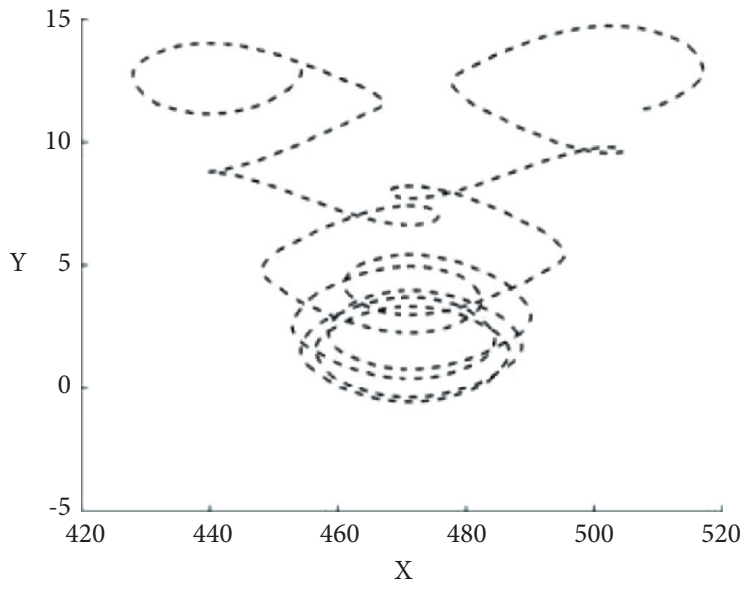

(a)

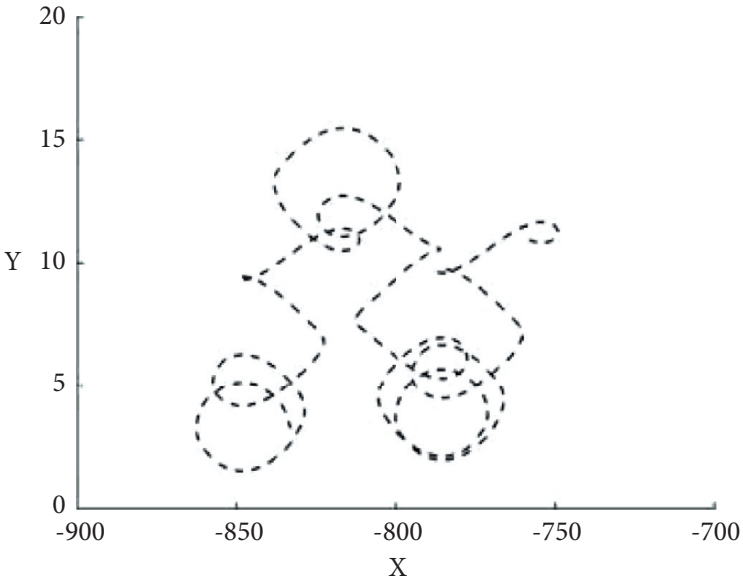

(b)

FIgURE 12: Continued. 


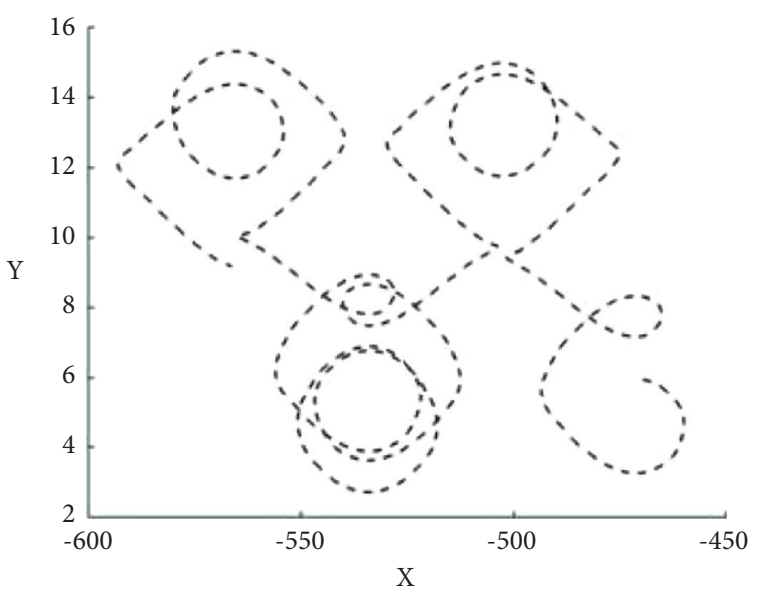

(c)

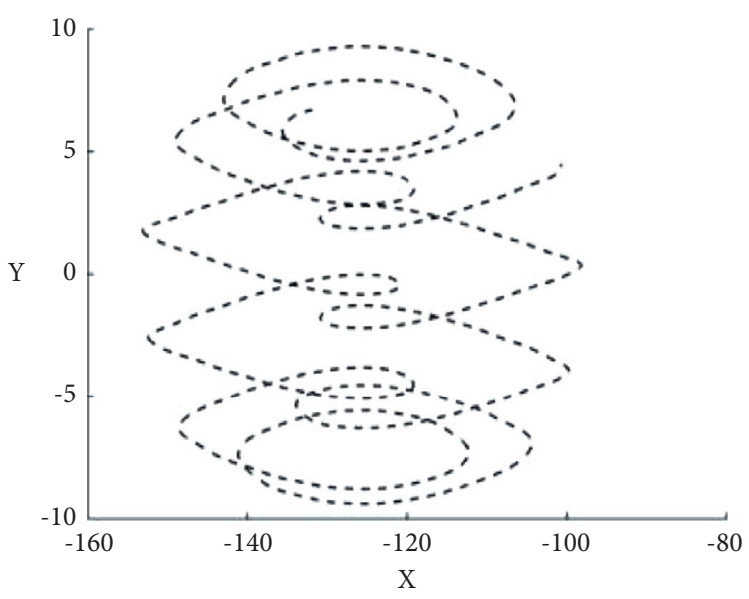

(d)

Figure 12: Phase portrait in the plane $(x, y)$ for System (2) illustrating megastability obtained by considering random initial conditions distributed around the $x$-axis. The frequency of the forcing term is $\omega=0.6$, and the amplitude $A=9.7$. Adding a forcing term can make the system exhibit various dynamics.

$$
\begin{aligned}
x_{n+1} & =x_{n}+h\left[-0.1 y_{n-1}+x_{n-1} \frac{\cos (r)}{r}\right] y_{n+1} \\
& =y_{n}+h\left[\sin \left(0.1 x_{n-1}\right)+y_{n-1} \frac{\cos (r)}{r}\right], \quad \text { where } r=\sqrt{x^{2}+y^{2}}, \\
x_{n+1} & =x_{n}+h\left[-0.1 y_{n-1}+x_{n-1} \frac{\cos (r)}{r}+A \sin (\omega t)\right] y_{n+1} \\
& =y_{n}+h\left[\sin \left(0.1 x_{n-1}\right)+y_{n-1} \frac{\cos (r)}{r}\right], \quad \text { where } r=\sqrt{x^{2}+y^{2}} .
\end{aligned}
$$

A system generator token is an important block that is dragged from the Xilinx block set library, which has information about the system generator model, through which it is possible to interface with the Vivado design tool to create an RTL design of the system. The phase planes of the proposed System (1) are shown in Figure 9, and the phase planes of System (2) are shown in Figures 10-12, which are obtained while running the system generator by changing initial conditions distributed around the $x$-axis.

\section{Conclusion}

A megastable system, the newest in the family of special chaotic systems, was designed and proposed. It was twodimensional flow coexisting attractors in some islands, interestingly covered by megalimit cycles. No equilibrium point was found for the proposed two-dimensional system. However, the origin $(0,0)$ acted like an unstable point. Adding a forcing term to the proposed system, chaotic solutions and coexisting strange attractors were obtained. Different behaviors were observed by altering the amplitude of the forcing term. Since the system was found to have no equilibrium point, the attractors were considered in the category of hidden attractors. The dynamical properties of this new system were investigated utilizing some tools such as attractor plots, bifurcation diagrams, and LEs diagrams. Two bifurcation and LEs diagrams were plotted to show the effect of initial conditions in the system's behaviors and dynamics. Phase portraits of the novel megastable oscillator were presented by FPGA design. Xilinx system generator block diagrams of the proposed system and trigonometric functions were also presented. The proposed system is a low-dimensional system with the ability to exhibit chaos by adding a forcing term. So, it can be used in some applications, such as a random number generator or image encrypting as future works.

\section{Data Availability}

All the numerical simulation parameters are mentioned in the respective text part, and there are no additional data requirements for the simulation results.

\section{Conflicts of Interest}

The authors declare that they have no conflicts of interest. 


\section{Acknowledgments}

The authors extend their gratitude to the Deanship of Scientific Research at King Khalid University for funding this work through the research group program under grant number R. G. P. 2/48/42.

\section{References}

[1] J. C. Sprott, "Some simple chaotic flows," Physical Review E, vol. 50, no. 2, pp. R647-R650, 1994.

[2] J. C. Sprott, Elegant Chaos: Algebraically Simple Chaotic Flows, World Scientific, Singapore, 2010.

[3] Q. Deng, C. Wang, and L. Yang, "Four-wing hidden attractors with one stable equilibrium point," International Journal of Bifurcation and Chaos, vol. 30, Article ID 2050086, 2020.

[4] J. C. Sprott, "Some simple chaotic jerk functions," American Journal of Physics, vol. 65, no. 6, pp. 537-543, 1997.

[5] C. Xu, J. Sun, and C. Wang, "An image encryption algorithm based on random walk and hyperchaotic systems," International Journal of Bifurcation and Chaos, vol. 30, Article ID 2050060, 2020.

[6] Q. Wan, Z. Zhou, W. Ji, C. Wang, and F. Yu, "Dynamic analysis and circuit realization of a novel no-equilibrium 5D memristive hyperchaotic system with hidden extreme multistability," Complexity, vol. 2020, Article ID 7106861, 16 pages, 2020.

[7] K. Rajagopal, M. E. Cimen, S. Jafari et al., "A family of circulant megastable chaotic oscillators, its application for the detection of a feeble signal and PID controller for time-delay systems by using chaotic SCA algorithm," Chaos, Solitons \& Fractals, vol. 148, Article ID 110992, 2021.

[8] T. Lu, C. Li, X. Wang, C. Tao, and Z. Liu, "A memristive chaotic system with offset-boostable conditional symmetry," The European Physical Journal Special Topics, vol. 229, no. 6-7, pp. 1059-1069, 2020.

[9] Z. Gu, C. Li, X. Pei, C. Tao, and Z. Liu, "A conditional symmetric memristive system with amplitude and frequency control," The European Physical Journal Special Topics, vol. 229, no. 6-7, pp. 1007-1019, 2020.

[10] Z. Wei, "Dynamical behaviors of a chaotic system with no equilibria," Physics Letters A, vol. 376, no. 2, pp. 102-108, 2011.

[11] M. Molaie, S. Jafari, J. C. Sprott, and S. M. R. Hashemi Golpayegani, "Simple chaotic flows with one stable equilibrium," International Journal of Bifurcation and Chaos, vol. 23, Article ID 1350188, 2013.

[12] A. N. Pisarchik and U. Feudel, "Control of multistability," Physics Reports, vol. 540, no. 4, pp. 167-218, 2014.

[13] H. Lin, C. Wang, and Y. Tan, "Hidden extreme multistability with hyperchaos and transient chaos in a hopfield neural network affected by electromagnetic radiation," Nonlinear Dynamics, vol. 99, no. 3, pp. 2369-2386, 2020.

[14] M. Chen, Y. Feng, H. Bao et al., "Hybrid state variable incremental integral for reconstructing extreme multistability in memristive jerk system with cubic nonlinearity," Complexity, vol. 2019, Article ID 8549472, 16 pages, 2019.

[15] M. Chen, M. Sun, H. Bao, Y. Hu, and B. Bao, "Flux-charge analysis of two-memristor-based chua's circuit: dimensionality decreasing model for detecting extreme multistability," IEEE Transactions on Industrial Electronics, vol. 67, pp. 2197-2206, 2019.

[16] J. C. Sprott, S. Jafari, A. J. M. Khalaf, and T. Kapitaniak, "Megastability: coexistence of a countable infinity of nested attractors in a periodically-forced oscillator with spatiallyperiodic damping," The European Physical Journal Special Topics, vol. 226, no. 9, pp. 1979-1985, 2017.

[17] Z. Wang, H. R. Abdolmohammadi, M. Chen et al., "A new megastable chaotic oscillator with singularity," The European Physical Journal Special Topics, vol. 229, no. 12-13, pp. 2341-2348, 2020.

[18] M. F. Danca, N. V. Kuznetsov, and G. Chen, "Approximating hidden chaotic attractors via parameter switching," Chaos: An Interdisciplinary Journal of Nonlinear Science, vol. 28, Article ID 013127, 2018.

[19] S. Cang, Y. Li, R. Zhang, and Z. Wang, "Hidden and selfexcited coexisting attractors in a lorenz-like system with two equilibrium points," Nonlinear Dynamics, vol. 95, no. 1, pp. 381-390, 2019.

[20] M. Chen, C. Wang, H. Bao et al., "Reconstitution for interpreting hidden dynamics with stable equilibrium point," Chaos, Solitons \& Fractals, vol. 140, Article ID 110188, 2020.

[21] G. A. Leonov, N. V. Kuznetsov, and T. N. Mokaev, "Homoclinic orbits, and self-excited and hidden attractors in a lorenz-like system describing convective fluid motion," The European Physical Journal Special Topics, vol. 224, no. 8, pp. 1421-1458, 2015.

[22] E. Tlelo-Cuautle, V. H. Carbajal-Gomez, P. J. Obeso-Rodelo, J. J. Rangel-Magdaleno, and J. C. Núñez-Pérez, "FPGA realization of a chaotic communication system applied to image processing," Nonlinear Dynamics, vol. 82, no. 4, pp. 1879-1892, 2015.

[23] H. Li, Z. Hua, H. Bao et al., "Two-dimensional memristive hyperchaotic maps and application in secure communication," IEEE Transactions on Industrial Electronics, vol. 68, no. 10, 2020.

[24] B. Bao, M. Peol, H. Bao et al., "No-argument memristive hyper-jerk system and its coexisting chaotic bubbles boosted by initial conditions," Chaos, Solitons \& Fractals, vol. 144, Article ID 110744, 2021.

[25] Q. Hong, H. Chen, J. Sun, and C. Wang, "Memristive circuit implementation of a self-repairing network based on biological astrocytes in robot application," IEEE transactions on neural networks and learning systems, no. 99, 2020.

[26] N. Tsafack, S. Sankar, B. Abd-El-Atty et al., "A new chaotic map with dynamic analysis and encryption application in internet of health things," IEEE Access, vol. 8, Article ID 137731, 44 pages, 2020.

[27] E. Tlelo-Cuautle, J. J. Rangel-Magdaleno, A. D. Pano-Azucena, P. J. Obeso-Rodelo, and J. C. Nuñez-Perez, "FPGA realization of multi-scroll chaotic oscillators," Communications in Nonlinear Science and Numerical Simulation, vol. 27, no. 1-3, pp. 66-80, 2015.

[28] A. Wolf, J. B. Swift, H. L. Swinney, and J. A. Vastano, "Determining lyapunov exponents from a time series," Physica D: Nonlinear Phenomena, vol. 16, no. 3, pp. 285-317, 1985.

[29] L. F. Ávalos-Ruiz, C. J. Zúñiga-Aguilar, J. F. Gómez-Aguilar, R. F. Escobar-Jiménez, and H. M. Romero-Ugalde, "FPGA implementation and control of chaotic systems involving the variable-order fractional operator with mittag-leffler law," Chaos, Solitons \& Fractals, vol. 115, pp. 177-189, 2018.

[30] H. Chen, S. He, A. D. Pano Azucena et al., "A multistable chaotic jerk system with coexisting and hidden attractors: dynamical and complexity analysis, FPGA-based realization, and chaos stabilization using a robust controller," Symmetry, vol. 12, no. 4, p. $569,2020$.

[31] K. Rajagopal, F. Nazarimehr, A. Karthikeyan, A. Srinivasan, and S. Jafari, "CAMO: self-excited and hidden chaotic flows," 
International Journal of Bifurcation and Chaos, vol. 29, no. 11, Article ID 1950143, 2019.

[32] A. Karthikeyan and K. Rajagopal, "FPGA implementation of fractional-order discrete memristor chaotic system and its commensurate and incommensurate synchronisations," Pramana, vol. 90, no. 1, p. 14, 2018.

[33] B. Karakaya, A. Gülten, and M. Frasca, "A true random bit generator based on a memristive chaotic circuit: analysis, design and FPGA implementation," Chaos, Solitons \& Fractals, vol. 119, pp. 143-149, 2019. 